ConNotas. Revista de Crítica y teoría literarias / Vol. VI, Núm. 10 / 2008

\title{
Ficción y memoria: narrador y estrategias discursivas en la literatura hispanoamericana contemporánea
}

\author{
SusAna InÉs GonZÁLEZ SAWCZUK*
}

Resumen:

Interesa la relación vincular de la ficción con la memoria que se manifiesta de acuerdo a las nuevas perspectivas de significación de lo real. Memoria que se construye por medio de estrategias discursivas que definen diferentes posicionamientos del narrador. Tres obras y tres escritores, en contextos diferentes, llevan adelante la exploración de los modos de narrar. Rodolfo Walsh (Argentina) deja estampada la memoria de una matanza en Operación masacre (1957), texto que combina la investigación y el periodismo y que se transforma en modelo de la ficción testimonial. En tiempos más cercanos, Elena Poniatowska (México) con estrategias diferentes recupera otro de los relatos del horror a partir de retazos de fragmentos, de breves crónicas de testigos, en La noche de Tlatelolco (1971). Por último, en 1980, se publica Respiración artificial (Ricardo Piglia). El personaje, Emilio Renzi, marca una narrativa de historias y significantes para leer otra dinámica política y cultural castigada por el terror y el exilio que trajo la última dictadura argentina de 1976.

\section{Palabras clave:}

Ficción y memoria, novela testimonial, estrategias narrativas, Ricardo Piglia, Elena Poniatowska, Rodolfo Walsh.

\footnotetext{
* Universidad Nacional de Colombia.
} 
En contextos marcados por la censura, la expresión literaria será un lugar con cierto resguardo y un campo de experimentación polifónica. Dentro de estas manifestaciones de la representación estética se encuadran la recuperación de la memoria y los usos y los efectos que provocan registros de la historia y que dan cuenta de la creación de una estrategia discursiva en la figura del narrador. Tres obras literarias marcadas por una escritura testimonial serán el punto de partida: Operación masacre (1957) de Rodolfo Walsh (Argentina), Respiración artificial (1980) de Ricardo Piglia (Argentina), y La noche de Tlatelolco (1971) de Elena Poniatowska (México). Son relatos que actualizan momentos históricos de fuerte impacto y descomposición social. El decir literario se transforma en un espacio de recuperación de significantes de lo social y de reconocimiento histórico. Son marcas de estos textos: el relato como investigación, la búsqueda del secreto, la verdad velada, la construcción de una narrativa marcada por los vacíos, fragmentos de testimonios, y por los mecanismos para eludir formas de censura.

La idea es observar la creación de una estrategia discursiva que recupere significantes y registros de la memoria. El foco está centrado en las diferentes situaciones de enunciación en que aparece el narrador. Si estamos en presencia del narrador omnisciente, el que focaliza todo, de un narrador que da entrada al testimonio, o ante otra variante, la del narrador que pasa a "frjarse" en un personaje: la focalización interna. ${ }^{1}$ La ficción hace posible la aprehensión de un mundo real, dentro del marco de enunciados lógicos - en consonancia con las reglas convencionales que permite el lenguaje escrito-, verosímiles y tan alejados de lo verdadero y de lo falso como amparados dentro de la instancia lúdica. Además, el autor empírico se somete al juego de esa dinámica textual porque, en ese mismo acto, no sólo se define y determina su rol social, sino que también otra

${ }^{1}$ Para el problema de los géneros discursivos y la teoría del enunciado parto de los siguientes autores: M. M. Bajtín (Estética de la creación verba), T. Todorov y M. M. Bakhtine (Le principe dialogique), G. Genette ("Discours du récit") y Mieke Bal (Teoria de la narrativa). 
instancia creada por la escritura, el narrador, ocupa su lugar. Bajtín nos aclara que "el autor-persona real está presente en la obra como una totalidad, pero nunca puede formar parte de la obra" (19 y 301). Es, como lo define, una naturaleza creadora y no creada. Para el desempeño del personaje en la ficción parto de la esencia misma de esta narrativa que al decir de Foucault es "el régimen del relato o [...] los diversos regímenes según los cuales (se relata): (la) posición del narrador [...]; (la) presencia o ausencia de una mirada neutra [...]; (el) compromiso de todo el relato en la perspectiva de un personaje o de muchos [...] o de ninguno en particular" (37). Para analizar el perfil y la caracterización del narrador en el universo de ficción parto del concepto de "perspectiva", 2 es decir, de la estrategia de situarse en "quién ve y cómo ve". Siempre dentro del marco de la ficción, la perspectiva es uno de los modos de regulación narrativa y tiene que ver con el lugar y la manera de la focalización, con el sujeto de la percepción. Por un lado, es necesario precisar dónde está colocado el punto de vista, el foco narrativo, y por otro, atender al estatuto del narrador.

Sabemos que ninguno de estos lugares de análisis son fijos, ni se dan de forma preponderante en el universo narrativo. Al contrario, ni el modo particular de esa visión narrativa, la perspectiva o la focalización de la narración, es uniforme, ni la otra instancia que atiende a quién habla es simple de establecer, ya que va más allá de la fijación del enunciado en la persona gramatical, y da lugar a pensar en los diferentes mecanismos de ocultación de la identidad del narrador. Por eso, más que quién ve, me interesa saber cómo es esa mirada.

${ }^{2}$ Véase Genette 206-207. Se debe recordar que se acepta el uso indistinto de perspectiva, focalización y punto de vista; si bien, este autor elige el término focalización. La focalización también se ubica entre otras de las formulaciones de Genette que han sido objeto de polémicas, por ejemplo la que despertó la inquietud de Mieke Bal y, además, las actualizaciones que realiza Genette en Nouveau discours du récit (Paris: Du Seuil, 1983). No se tendrán en cuenta las diferencias de apreciación ya que exceden el interés de esta presentación. 
Otra de las lecturas que se abren atiende a la preocupación de la relación que se establece entre la ficción y la verdad. Y en ese matiz indecible entre la verdad y la falsedad se juega todo el efecto de la ficción. Se puede pensar la instancia que instala un relato de otra forma de significado que al decir de Barthes se podría llamar "ilusión referencial", donde la categoría de lo real emerge en detalles, y no en sus contenidos contingentes. En la singularidad de la descripción se refuerza un discurso realista. Se recupera el sentido de efecto de verdad que puede lograr el relato ficticio, al provocar una suerte de ilusión de convención de verdad o de instalación de lo verosímil. ${ }^{3}$ En la ficción, además, todos los cruzamientos son posibles, la política, la historia, lo público, lo privado y las creencias. El relatar aparece así como una forma de componer lo real, de volver a restituir con palabras la verdad de los hechos.

\section{Periodismo y literatura: hablan los testigos}

El sentido que guía esta reflexión se condensa en ¿cómo narrar y cómo significar lo indecible? ¿Cómo hay que contar el horror? Hasta dónde el artificio del lenguaje, el artilugio de cualquier (re)presentación hace posible recuperar una historia de dolor. Volver a contar parecería que permite acceder al lugar seguro del entendimiento al transmitir un relato verosímil en ese juego irreal de temporalidad que instaura toda ficción. Dos textos de dos escrituras distantes en tiempo y espacio ponen en escena realidades trágicas. En 1957, el escritor Rodolfo Walsh recopila y da forma de relato a las investigaciones que había publicado en el periódico Mayoría. Nace Operación masacre (1957) que rescata la memoria de un fusilamiento clandestino (en junio de 1956 un grupo de civiles

${ }^{3}$ Como se interpreta de la obra de Aristóteles, la verosimilitud que expresa semejanza con lo verdadero, la apariencia, donde las cosas parecen ser y no son. "No corresponde al poeta decir lo que ha sucedido sino lo que podría suceder, esto es, lo posible según la verosimilitud o la necesidad” (157). Véase capítulos 9, 24 y 25. 
son secuestrados por fuerzas policiales y serán fusilados en un descampado de la Provincia de Buenos Aires, en José León Suárez). Eran tiempos de dictadura. Un golpe militar derroca al gobierno peronista en 1955 y establece un gobierno provisional con los generales Aramburu y Rojas. En junio de 1956, fuerzas militares que respaldan al peronismo se levantan, al mando de los generales Valle y Tanco, reprimidos y también fusilados. Operación masacre se encuadra en este contexto y "es el prólogo de la tragedia que vendrá después. Aramburu y Rojas serán el prólogo de Videla y Massera” (Bayer 11).

El autor entra al texto, advierte y deja claro su posicionamiento. En la presentación (prólogo), dos breves frases condensan cómo se entera de esa historia y qué hará con ella. En un bar, alguien le dice: "Hay un fusilado que vive" (Walsh 19). Y para Rodolfo Walsh, el escritor de cuentos policiales, el periodista, el traductor, apasionado del ajedrez y de la literatura fantástica, ese dato es suficiente y "Así nace aquella investigación, este libro" (19). El perfil y la perspectiva del narrador es la del periodista-investigador. Un mediador que da lugar y pone en escena a los testigos. La obra tiene tres partes: Las personas, Los hechos y La evidencia. Tres momentos que arman un relato, una investigación que va a demostrar y a sacar a luz la verdad. Dos aspectos confluyen para reflexionar acerca de la ficción que ocupa el lugar del testimonio. El primero es la escritura, las poéticas o los discursos que están puestos en juego, y que se sitúan entre dos dinámicas: entre la literatura y el periodismo o entre la ficción y la crónica; y una búsqueda que guía al narrador y sobre determina su lugar en el relato: la verdad. Y, el segundo aspecto es el lugar que elige para contar y el desplazamiento que realiza el narrador.

En la primera parte, Las personas, el narrador reconstruye historias de vida, gestos, momentos, es un narrador neutral, omnisciente, casi un presentador impecable de una escena que adelanta y vislumbra el terror y la tragedia que espera a esos personajes. Sin embargo, va dejando pistas, datos inconclusos, alusiones, misterios. Como en los relatos policiales se reconstruye la escena previa, como en toda buena reconstrucción de un crimen por venir. En los 
apartados siguientes, el narrador es el periodista que investiga y que presenta las voces de los testigos, las versiones que se contradicen y confunden pero sólo con la fuerza del testimonio es posible hacer aparecer la verdad ya que la construcción de la ficción "es antagónica con la estética urgente del compromiso" (Piglia 14), por eso el desplazamiento del discurso hacia las otras voces. Y es en los detalles en los que se encamina el descubrimiento de la verdad, en las conjeturas, en esas intervenciones del narrador que, en las notas al pie, estructura juicios y apreciaciones para constatar los hechos. El testimonio es un relato que debe ser interpretado pero que tiene la fuerza de recuperar esa "temporalidad originaria" (Ricoeur).

En esa suma de versiones, el narrador-periodista-detective reconstruye un crimen, una masacre escondida y negada por el poder institucional y por las fuerzas represivas, y recupera para la memoria social un momento de la historia argentina. Ese desplazamiento "de no decir y hacer decir a los otros", esa combinación de técnicas "conjetural y perspectivismo" (Romano 82-97), en fin, esa posición que ocupa el narrador y esa elección de dar lugar al testimonio lo define, porque:

El testigo certifica la verdad y permite que el que escribe vea la escena y pueda narrarla, como si fuera otro. Ir hacia otro, hacer que el otro diga la verdad de lo que siente y de lo que ha sucedido, ese desplazamiento, este cambio en la enunciación, funciona como un condensador de la experiencia. (Piglia, "Tres propuestas" 18)

El relato de otra matanza quedará estampado en La noche de Tlatelolco, otro texto que, como Operación masacre, continúa editándose. La escritora Elena Poniatowska elige al testimonio como el único régimen del relato apto para recuperar la verdad de una historia que ha sido negada. El texto está organizado en dos partes y una cronología final: "Primera parte. Ganar la calle" y "Segunda parte. La noche de Tlatelolco". El corpus textual es un collage, una suma de pequeños fragmentos, testimonios de estudiantes, maestros, obreros, comerciantes, de la prensa escrita, de algunos granaderos y va- 
rias madres y otras voces del pueblo. Son micro historias que sugieren su correlato, y se van hilvanando por el sentido cronológico que define la estructura del texto, en esas dos partes diferentes en tiempo e intensidad de los hechos.

A diferencia de Operación masacre, la presencia de un narrador es casi inexistente y se confunde, en este caso, con la autora empírica. En La noche de Tlatelolco, una suma de fragmentos, de frases entrecortadas, de crónicas dan continuidad y mantienen la tensión de una historia que se vislumbra trágica. Sin embargo, sabemos que además de la presencia oculta de una escucha, de una cronistaperiodista que recopiló los relatos, que los seleccionó, que separó los tiempos y las voces y definió una edición, las tres intervenciones que realiza la autora refuerzan y recuerdan que alguien está guiando y dando curso a esa escritura.

La primera intervención es la entrada al texto (La noche 13-14); no hay prólogo ni introducción. Descriptiva y concisa, es el relato de una visión: la multitud de jóvenes marchando. Estos breves párrafos funcionan como una sinécdoque del horror de Tlatelolco, y esta primera visión parecería ocupar el primer testimonio de una primera testigo, lugar que elige quien narra para fijar en el texto y en la memoria de cada lector la verdad de la historia que ha sido silenciada. Por eso, "E. P.", que se presenta como otra de las voces, como otro de los múltiples testigos es, sin embargo, quien puede sintetizar, quien puede condensar los testimonios en un único relato inicial que tiene la forma de una aparición, especie de epifanía que adelanta el horror. Las siguientes dos intervenciones de E. P. se dan al comienzo de la segunda parte (164 y 166-171) pero, por la gravedad del relato que adelanta el desenlace fatal, la posición de testigo asumida anteriormente se modifica. Hay una breve presentación de la crónica de esos días, advertencias, y casi una apelación para recordarnos siempre que "este relato les pertenece" a otros. Elena Poniatowska parece responder mejor que nadie a la pregunta que guía esta presentación. No se puede contar el horror, porque "El dolor es un acto absolutamente solitario. Hablar de él resulta casi intolerable; indagar, horadar, tiene sabor de insolencia" (164). 
Por último, elegir el testimonio, el relato en fragmentos, dando lugar a la denuncia es la forma de dar cuenta de la verdad. Aunque esas múltiples escrituras subjetivas a veces contradictorias, de signos y significantes en exceso sigan siendo escrituras despersonalizadas ya que los nombres no nos dicen nada, no está presente el juicio mediático de la figura fuerte de un narrador periodista (como en Operación masacre). Ante este otro horror, quien narra se retrae. La verdad está puesta en el relato crudo.

\section{Cómo contar la dictadura argentina}

La publicación en 1980 de Respiración artificial tiene una significación particular que trasciende, en Argentina, el ámbito cultural. Además de la crítica literaria, desde el campo de la historia y de la sociología ${ }^{4}$ se coincide en destacar a esa fecha como una especie de bisagra político-social que da lugar a una serie de manifestaciones para recuperar una dinámica política y cultural castigada, silenciada y fragmentada por el terror y el exilio. Como antecedente y corolario del año 80, se recuerda, además, la llegada al país en 1979 de la CIDH que impulsa y da validación a la lucha de las nuevas organizaciones de defensa de los derechos humanos; y la crisis institucional que afecta la cohesión de las FFAA, expresada en los recambios de mando que culmina en 1982 con el poder en la figura de Galtieri,

\footnotetext{
${ }^{4}$ Ver, entre otros: Saúl Sosnowski (comp.), Represión y reconstrucción de una cultura: el caso argentino. (Buenos Aires, EUDEBA: 1988); José Amícola, "La literatura argentina desde 1980: nuevos proyectos narrativos después de las desapariciones de Cortázar, Borges y Puig" (Revista Iberoamericana. Pittsburg univ. USA. Vol. LXII. N 175. Abriljunio 1996: 427-438); A. Avellaneda, "Best-Sellers y código represivo en la narrativa argentina del ochenta: el caso Asís” (Revista Iberoamericana. Número especial dirigido por Silvia Molloy. Pittsburg univ. USA. Vol. XLIX. Núm 125. Octubre-dic. 1983: $983-$ 996); Ester Azubel, "Narrar la experiencia: notas acerca de la literatura argentina de los 80" (Reflejos. Depto. de Estudios españoles y latinoamericanos. ARLO. Israel. Universidad Hebrea de Jerusalén, núm 4. Diciembre de 1996: 8-17); Tulio Halperin Donghi. El espejo de la historia. (Buenos Aires: Sudamericana, 1987).
} 
quien acelera la caída de un régimen de terror con la Guerra de las Malvinas. Es a partir de esta fecha que se pueden encontrar los puntos de inflexión de un sistema autoritario que dejó una sociedad marcada por el terror y con la necesidad de garantizar espacios de reflexión. Es, entonces, en 1980 que aparece la primer novela de Ricardo Piglia.

Si en los años previos la literatura argentina se leía en el exilio, es decir en México, en España o en París, entre otros, un fenómeno editorial $^{5}$ daba cuenta de otra relación de la ficción con la realidad, de otros modos de representación del decir literario y artístico en general, que se podrían sintetizar en la exploración de manifestaciones para dar respuesta a la cuestión de cómo significar, cómo narrar. En los ochenta el debate cultural penetra en una sociedad expectante por encontrar sentidos a una experiencia dolorosa. La obra Respiración artificial no puede ser pensada fuera de este contexto, pero interesa también la apropiación que se hace de la novela y del efecto que produce en los lectores, como señala Halperin Donghi:

No es exagerado, pero sí paradójico, calificar de clamoroso el éxito de esta novela de Ricardo Piglia, que no logró agotar en largos años su primera edición [...], logró encontrar lectores que en la historia allí narrada reconocieron la suya propia, y si esos lectores eran menos numerosos [...], no eran por eso menos capaces de crear un clima de opinión. (80)

Parecería que una suerte de efecto retardatario, como consecuencia de ese doble movimiento que produce la lectura, "comprender e

${ }^{5}$ En 1980, además de Respiración artificial, se publican Flores robadas en los jardines de Quilmes, de Jorge Asís y Juanamanuela mucha mujer, de Martha Mercader. Sirve esta muestra de registros tan diferentes, como señala Jorge Lafforgue, porque "quebró una constante cultural instalada al calor de la política económica de Martínez de Hoz. [...] el predio de los best-sellers pertenecía exclusivamente a connotados escritores extranjeros". La novela de Asís, por otro lado la de mayor éxito de mercado "que hacía referencia nada encubierta a los años de represión más dura, [...] clausuró el período del debate subterráneo, sacando a luz el latente interés de un público vasto por los temas nacionales" (Sosnowski 153). 
incorporar", sigue acompañando a Respiración artificial que continúa editándose. Novela que encuentra lectores que se reconocen en ese relato, lectores que ponen en juego y recuperan en esa lectura la re-escritura de experiencias, historias y relatos privados. Es esta obra la que posiciona a Piglia en la narrativa hispanoamericana y lo convierte en uno de los principales y más editados escritores argentinos, y es la que cuenta con las mayores investigaciones académicas e interpretaciones críticas.

En síntesis, Respiración artificial abre una variedad de lecturas. Es una obra plagada de recursos retóricos. La intertextualidad se exacerba como modalidad narrativa, se construye sobre citas, plagios, recitados, alusiones, dando lugar a una auténtica ficción-crítica (Rosa). Es una novela de exploración, que mezcla diferentes registros en el discurso: la epístola, el documento histórico, el diario, el archivo. Es la obra, al momento, que condensa la trayectoria de nuestro escritor. Se puede leer dentro del marco de las ficciones de búsqueda, como un espacio de recuperación de los sentidos históricos. Contra las interpretaciones totalizadoras, opone el presente en clave del pasado; contra una temporalidad lineal, el desplazamiento y la alteración cronológica atraviesan el relato. Se puede, también, leer como un relato de investigación policial, que cumple las pautas esperadas del género; develar el secreto y encontrar el sentido del fracaso histórico requiere un lector cómplice, atento, que tenga competencias culturales y sepa entender las pistas. En fin, novela híbrida, búsqueda de la verdad, interrogación de la historia, ficción utópica o ensayo ficcionalizado, todas estas son lecturas que se disparan de la obra; y son todas estas y otras más que se hicieron de Respiración artificial.

En las tres primeras líneas se manifiesta la transparencia de un narrador que, a pesar de los desplazamientos, interrupciones y confusiones, es el eje del relato. Este narrador es Emilio Renzi. El narrador se pregunta: ¿Hay una historia? Si hay una historia empieza hace tres años. En abril de 1976, cuando se publica mi primer libro, él me manda una carta (Piglia, Respiración 13). Abril de 1976 es demasiado alusivo, menciona los días posteriores al 24 de marzo de 1976, fecha en que se produce el último golpe militar en Argentina 
que inauguró los años del terror: el terrorismo de estado. En principio, sabemos en qué lugar y en qué tiempo está ubicado, en Argentina y en 1979. Sabemos también que es escritor (publicó su primer libro en abril de 1976) y que puede tener aproximadamente 39 años (13). Y se verá que no hay una, sino varias historias que difuminan la escritura en un caos cronológico; se trazan paralelos de vida, se buscan verdades en un pasado histórico, en secretos de familia (con Luciano Ossorio, Esperancita) se exploran archivos, cartas que puedan resolver el enigma de un suicida Enrique Ossorio, posible traidor o patriota, que vivió en el siglo XIX, en plenas guerras civiles. Y del otro lado, alejado en un pueblo de Entre Ríos, en un presente suspendido, está Marcelo Maggi, tío de Emilio. Los une, además de una relación epistolar, un sentido de la dimensión histórica del horror. Como señala Daniel Balderston: "el libro está hecho de (los) intentos orales por expresar la verdad pero nunca la verdad enteramente revelada" (12). Hay que buscar, despejando la densidad de las palabras pero enfrentándose a espacios vacíos.

Respiración artificial se divide en dos partes que diferencian dos espacios de la intriga, con nuevos personajes y con otro sujeto narrativo. Esta división separa, para Renzi, comportamientos distintos en esos espacios de contención. En la primera parte, el personaje Renzi se apropia del foco, ajusta la mirada, es más reflexivo, más testigo que protagonista. En cambio, en la segunda parte, Renzi casi no tiene voz en la narración no es el principal narrador, sin embargo, sus intervenciones tienen un valor, hace uso de un discurso provocador, especula y disputa una autoridad intelectual. En la apertura del relato se instala el dilema que guía la novela, que se resuelve en ¿cómo narrar? Emilio Renzi es quien lleva adelante la exploración de los modos de narrar.

Se narra una historia privada, una biografía con las historias de los otros. Se pasa del ámbito privado a las entrañas históricas: cartas, fotos, historias familiares se entrelazan con documentos, con fragmentos de testimonios, biografías, restos de informes secretos para dar lugar a un discurso que sostiene un secreto que hay que revelar, y que se arma de fragmentos. Interesa observar el contraste que descubre el texto ante un narrador, Emilio Renzi, definido en 
tiempo y espacio, con características delimitadas, con una biografía conocida, que interactúa en un juego de alteración de la temporalidad con personajes que vienen del pasado, que se ubican en un futuro y que hasta están en un "un presente sin presencia" (Romano 279) como su tío Marcelo Maggi. Novela hecha de citas, (De Grandis) texto disparador de historias y de personajes fuera de lugar. Este desplazamiento que realiza Renzi, en el tiempo y sobre textos, es una marca que define la obra. Todos los demás personajes también están dislocados o buscan algo que está en otro sitio y hasta en otro tiempo (Marcelo Maggi, Enrique Ossorio); y se definen por esa traslación o están en un sitio y lugar que no corresponde al esperado (Luciano Ossorio, Tardevsky). El relato es una suma de condensaciones que se disparan y abren enunciados donde nunca se dice lo que se tiene que decir, de la forma que se espera que se diga.

En el apartado II: la memoria es el eje que concentra la tensión de la novela. Este tratamiento tiene lugar en la historia del senador Luciano Osorio. La relación vincular de la ficción con la memoria se manifiesta de acuerdo a las nuevas perspectivas de significación de lo real de muchas novelas actuales donde, como señala Susana Zanetti:

la Historia se transforma en memoria y esta memoria se construye a partir de la mezcla de voces y discursos, de restos, de fragmentos que dan nueva dimensión a lo anónimo y colectivo de los sujetos [...]. La fragmentación o la dispersión del acontecer histórico o de sus actores es una de las perspectivas fundamentales [...]; la otra, es la de la conjetura. Quien enuncia interpreta o fabula, conjetura, cede la palabra o se desdobla, asume diversos lenguajes. (10)

Ambas perspectivas confluyen en Luciano Ossorio que representa él mismo la memoria y enlaza su genealogía con la Historia. Es testigo y su desesperación está en llegar a la situación de no poder prestar testimonio, "en llegar a perder el uso de la palabra". "Llegar a concebirla, [...], y no poder expresarla" (Piglia, Respiración 
47). En estas pocas páginas se delimita el clímax de la ficción que enlaza dos fuerzas contrapuestas. Por un lado, el exceso de palabras que se manifiesta en la abundancia de relatos, historias, testimonios, biografías, cartas, documentos que recorren toda la novela; y por otro, el vacío que define la alocución de Ossorio ante la posibilidad de la pérdida de la expresión en sus distintas formas. Emilio Renzi es el elegido para ser testigo. Él es el único que se conecta con casi todos (excepto con Marcelo), porque todos tienen una parte que puede completar aquello que busca. Es el que desea enterarse, para contar; y eso es el historiador: testigo para dar testimonio. Si bien el principal narrador es otro personaje, Tardewski, ${ }^{6}$ su interlocutor es Emilio.

En síntesis, en el primer tramo de la novela, el narrador tiene una actitud de movilidad constante, sus características adolecen de seguridad. Se confunde e interrumpe su voz con facilidad, es antes que nada un narrador que se desorganiza y desorganiza los enunciados, las voces y las anécdotas del relato. Ahora, en cambio, en esta segunda parte, Renzi, más que dispersar, condensa. Más que abrir instancias caóticas del relato, sobresale su intento por organizar, por ordenar. Se habla de literatura, se acumulan hipótesis acerca de autores y obras, se pone en movimiento un discurso provocador, se observa, en síntesis, un despliegue de apreciaciones ya conocidas de Ricardo Piglia, sobre la formación de la tradición literaria nacional. ${ }^{7}$ En esta última instancia del texto se explora la dimensión filo-

${ }^{6}$ Intelectual polaco, formado en filosofia, amigo de Marcelo Maggi, cuya historia de vida tiene fuertes asociaciones con Witold Gombrowicz. Sin embargo, en el texto queda claro que no es la misma persona, dice Tardewski "Yo era el último de una lista que se iniciaba [...], con Pedro de Angelis y llegaba hasta mi compatriota Witold Gombrowicz" (Piglia, Respiración 110).

${ }^{7}$ Básicamente, se repiten aquí las tesis conocidas de nuestro escritor acerca de la importancia de la línea europea en la formación de una tradición literaria argentina, del modelo del intelectual nacional, de las parejas que definen una tradición, por ejemplo Borges-Groussac, Borges-Arlt, etc., y la frase, casi histórica, de Renzi: “(Borges) es el mejor escritor argentino del siglo XIX” (Piglia, Respiración 127 y 110-142). 
sófica, literaria y política para concluir que la verdadera función del conocimiento es siempre destructiva (153). Más que en la desolación, es en el silencio de la soledad (ausencia de sentido al que arribó el recorrido de una filosofía racional) donde se encuentra la respuesta a cómo narrar lo indecible.

Este itinerario intelectual trasciende la identidad nacional y al modo de cumplir un designio teleológico encuentra, en el recorrido de la comprensión y el conocimiento, formas inacabadas que no alcanzan para evitar el horror. ${ }^{8}$ Se recuerda que en esta parte se enlaza una cosmovisión que abarca desde el concepto formalista de la "ostranenie", del uso que hace del mismo Bertolt Brecht," pasando por el movimiento filosófico del Círculo de Viena, hasta confluir con el simbólico encuentro/enfrentamiento Kafka-Hitler, para culminar con la aseveración dramática de un inevitable desenlace destructivo del conocimiento. En Respiración artificial las intrigas se entrecruzan, no hay una resolución final, no hay lugares de

${ }^{8}$ En el trazado de este itinerario se observa una ligazón fuerte con el pensamiento de T. Adorno y M. Horkheimer en Dialéctica del iluminismo (1947), obra cumbre, inseparable del contexto de producción de la trágica experiencia de la Segunda Guerra, que formula el fin de las expectativas de confianza en el desarrollo progresivo de una racionalidad. Los autores invierten la hipótesis y demuestran cómo, precisamente, el desarrollo de la razón del iluminismo llevó a esta nueva barbarie.

${ }^{9}$ Se destaca en estos párrafos el despliegue que el narrador realiza de referencias puntuales de aportes críticos, que van más allá de una puesta en cuestión y de un uso de la crítica en el espacio de ficción, y que remiten a una especificidad demasiado excluyente. Por ejemplo, señalar la diferencia de aprehensión que realiza B. Brecht del concepto de "ostranenie", quien lo adapta y lo incorpora "como efecto de ruptura de la ilusión o distanciamiento", entre otras razones, para conmover, provocar y sacar de la pasividad al espectador a diferencia del texto primordial de este concepto, "El arte como artificio" (1916-17), de Shklovsky. Éste encuentra en una nueva lectura de Tolstoi el sentido artístico de un texto, a partir del descubrimiento de un procedimiento: "la ostranenie" (el extrañamiento. Se aclara que la versión española -que lo toma de la francesa- lo traduce, en cambio, como "singularización". Ver Amícola, De la forma). El procedimiento del arte, la ostranenie, es un modo diferente de observar la realidad, un cambio de percepción. Es éste un aporte fundamental del pensamiento formalista ruso. Ver T. Todorov. Teoría de la literatura de los formalisas rusos y José Amícola. De la forma a la información, 58 y ss. 
arribo, hay desvíos, partidas hacia diferentes puntos. Y Emilio Renzi es quien va a desandar en la novela las diferentes vertientes para contar, para narrar.

Por último, Operación masacre, La noche de Tlatelolco y Respiración artificial son textos que tienen en común la oralidad como principio ordenador que estructura los múltiples relatos. Al dar lugar a la palabra de los otros, a los testigos, y hacer uso de un discurso dislocado "para hablar de lo que no se puede hablar", desde otro lugar, se coloca al testimonio como verdad y no como representación. Y en esos desplazamientos de discursos que atestiguan (La noche de Tlatelolco), de documentos y pruebas de un narrador que investiga y saca a luz la verdad (Rodolfo Walsh) o de la exacerbación de relatos y caos temporal y espacial de Respiración artificial, operan diálogos y traslados discursivos que a menudo la literatura establece con las ciencias sociales, como lugares de intercambio entre la ficción y la política, entre la historia y la imaginación.

\section{Bibliografía}

Altamirano, Carlos y Beatriz Sarlo. Literatura/Sociedad. Buenos Aires: Hachette, 1983.

Amícola, José. "La literatura argentina desde 1980: nuevos proyectos narrativos después de las desapariciones de Cortázar, Borges y Puig." Revista Iberoamericana 42.175 (1996): 427-438.

- De la forma a la información: Bajtín y Lotman en el debate con el formalismo ruso. Buenos Aires: Beatriz Viterbo, 1997.

Aristóteles. Poética de Aristóteles. Edición trilingüe a cargo de Valentín García Yebra. Madrid: Gredos, 1974.

Avellaneda, Andrés. “' 'Best-Seller' y código represivo en la narrativa argentina del ochenta: el caso Asís." Revista Iberoamericana 49.125 (1983): 983-996.

Azubel, Ester. "Narrar la experiencia: notas acerca de la literatura argentina de los 80.” Reflejos 4 (1996): 8-17.

Bal, Mieke. Teoria de la narrativa. Madrid: Cátedra, 2006. 
Balderston, Daniel. Ficción y Política. La narrativa argentina durante el proceso militar. Buenos Aires: Alianza, 1987.

Bajtín, M. M. Estética de la creación verbal. Tr. Tatiana Bubnova. México: Siglo XXI, 1989.

Barthes, R. "O efeito de Real.” Literatura e Semiologia. Por G. Genette, T. Todorov, et al. Tr. Célia Neves Dourado. Petrópolis. Río de Janeiro: Ed. Vozes Ltda, 1972. 35-44.

Bergero, Adriana y Fernando Reati, comps. Memoria colectiva y políticas de olvido. Argentina y Uruguay, 1970-1990. Buenos Aires: Beatriz Viterbo, 1997.

Brunner, Joaquín J. y A. Flisfisch. Los intelectuales y las instituciones de la cultura. Santiago de Chile: FLACSO, 1988.

De Grandis, Rita. "La cita como estrategia narrativa en Respiración artificial." Revista Canadiense de Estudios hispánicos 8.2 (1993): 259269.

Foucault, M. "La proto-fábula." Verne: un revolucionario subterráneo. En AAVV. Buenos Aires: Paidos, 1968.

Genette, G. "Discours du récit." Figuras III. Paris: Du Seuil, 1972. Halperin Donghi, Tulio. El espejo de la historia. Buenos Aires: Sudamericana, 1987.

Lafforgue, Jorge, ed. Textos de y sobre Rodolfo Walsh. Buenos Aires: Alianza, 2000.

Orlandi Eni, P. As formas do silencio: no movimento dos sentidos. Sao Paulo: Ed. UNICAMP, 1997.

Piglia, Ricardo. Critica y ficción. Buenos Aires: Siglo veinte, 1993. . Respiración artificial (1980). Buenos Aires: Sudamericana, 1993.

."Tres propuestas para el próximo milenio (y cinco dificultades)." Casa de las Américas 222 (2001): 11-21.

Pizarro, Ana, coord. América Latina: Palavras, literatura e cultura. Vol. 3. Sao Paulo: Memorial UNICAMP, 1995.

Poniatowska, Elena. La noche de Tlatelolco. México: Ediciones Era, 1974.

. Fuerte es el silencio. México: Ediciones Era, 1987.

Reati, Fernando. Nombrar lo innombrable. Violencia política y novela argentina: 1975-1985. Buenos Aires: Legasa, 1992. 
Ricoeur, Paul. Texto, testimonio y narración. Tr., pról. y notas de Victoria Undurraga. Santiago de Chile: Editorial Andrés Bello, 1983.

Romano, Evelia. "Marcelo: un presente sin presencia en Respiración artificial de Ricardo Piglia." Sobretiro. Nueva Revista de Filología Hispánica 41.1 (1993): 279-291.

Rosa, Nicolás. "Veinte años después o la 'novela familiar' de la crítica literaria." Cuadernos hispanoamericanos 517-519. (1993): 161-186.

Sosnowski, Saúl, et al., comps. Represión y reconstrucción de una cultura: El caso argentino. Buenos Aires: EUDEBA, 1988.

Todorov, T. Teoría de la literatura de los formalistas rusos. Buenos Aires: Signos, 1979.

. y M. Bakhtine. Le principe dialogique. Paris: Du Senil, 1981.

Valenzuela, Luisa. "Simetrías." Cuentos de historia Argentina. Selección y prólogo Guillermo Saavedra. Buenos Aires: Alfaguara, 1998.

Walsh, Rodolfo. Operación masacre. Buenos Aires: Ediciones de la Flor, 2000.

Zanetti, Susana. "Historia y ficción en la novela hispanoamericana contemporánea." Revista de Lengua y Literatura. (Universidad Nacional del Comahue) 1.2 (1987): 4-14. 